\title{
La relación de la toma de decisiones y la gestión educativa en docentes gestores de la universidad
}

\section{The relationship of decision-making and educational management in teachers managers of the university}

Judith Soledad Yangali Vicente Universidad de Cañete, Perú

José Luis Rodríguez López

Universidad Cesar Vallejo, Perú

Melba Rita Vásquez Tomás

José Gabriel Chahuara Ardiles

Universidad Santo Domingo de Guzmán, Perú

Autor para correspondencia: judithsyv@gmail.com,rvasquez_25@hotmail.com, luisjoserodriguez1512@gmail.com,jgchahuara@gmail.com

Fecha de recepción: 23 de agosto de 2018 - Fecha de aceptación: 01 octubre de 2018

Resúmen: La investigación tuvo como objetivo determinar la relación directa entre la toma de decisiones y la gestión educativa en docentes de la universidad privada Sergio Bernales. En la gestión educativa la toma de decisiones, es un factor determinante en la resolución de problemas, el líder pedagógico, es el docente gestor, que asume funciones inherentes a la gestión, asi como acciones vinculadas a la responsabilidad social universitaria y la investigación; quien, a su vez, es el responsable de la toma de decisiones, para alcanzar los objetivos institucionales. La investigación fue de tipo aplicada, con un diseño no experimental, de corte transversal y enfoque cuantitativo. El método de análisis de datos utilizado, fue la correlación de Pearson. La población se constituyó de 84 docentes universitarios de las carreras de ingeniería de Sistemas, obstetricia y derecho y ciencia política de la universidad privada Sergio Bernales, ubicado en la provincia de Cañete, Lima. El instrumento de recolección de datos fue una lista de cotejo aplicada a una muestra de 42 docentes. Concluyéndose que existe una relación directa entre la toma de decisiones y la gestión educativa en los docentes de la universidad privada Sergio Bernales.

Palabras Claves: líder pedagógico; universitarios; resolución de problemas

Abstract: The research aimed to determine the direct relationship between decision making and educational management in teachers of the private university Sergio Bernales. In educational management, decision-making is a determining factor in the resolution of problems, the pedagogical leader is the managing teacher, who assumes functions inherent to management, as well as actions linked to university social responsibility and research; who is responsible for making decisions, to achieve institutional objectives. The research was of the type applied with a non-experimental, cross-sectional design and quantitative approach. The data analysis method used was the Pearson correlation. The population was made up of 84 university professors from the systems engineering, obstetrics and law and political science courses at the private university Sergio Bernales, located in the province of Cañete, Lima. The data collection instrument was a 
checklist applied to a sample of 42 teachers. Concluding that there is a direct relationship between decision making and educational management in the teachers of the private university Sergio Bernales.

Key Words: pedagogical leader; university students; problem solving

\section{Introducción}

\section{La gestión educativa}

De acuerdo con Casassus (2000) "La teoría de la gestión es la comprensión e interpretación de los procesos de la acción humana en una organización” (p. 13). La gestión educativa es entendida como el conjunto de actividades y diligencias estratégicas guiadas por procedimientos y técnicas adecuadas para facilitar que las instituciones logren sus metas, objetivos y fines educacionales mientras que la toma de decisiones es una acción donde la persona está expuesta en todo momento de su vida y para ello debe contar con los elementos e insumos necesarios que le permita dar las alternativas favorables frente a una situación problemática que presenta la organización. De acuerdo con Chadwick (1978) "la gestión educativa como aspecto fundamental de la educación juega un rol importante en la conducción y realización de las actividades, que van a conducir al logro de las metas y objetivos previstos en el sistema educativo" (p. 60).

\section{Problema general}

¿Cuàl es la relación directa entre toma de decisiones y la gestión educativa en los docentes de la universidad privada Sergio Bernales?

\section{Problemas específicos}

- ¿Cuál es la relación directa entre las dimensiones decisión oportuna y gestión institucional en los docentes de la universidad privada Sergio Bernales?

- ¿Cuál es la relación directa entre las dimensiones análisis de información y la gestión pedagógica en los docentes de la universidad privada Sergio Bernales?

- ¿Cuál es la relación directa entre las dimensiones flexibilidad y adaptación y gestión comunitaria en los docentes de la universidad privada Sergio Bernales?

- ¿Cuál es la relación directa entre las dimensiones evaluación de alternativas y gestión

- ¿Administrativa en los docentes de la universidad privada Sergio Bernales?

\section{Objetivo general}

Determinar la relación directa entre la toma de decisiones y la gestión educativa en los docentes de la universidad privada Sergio Bernales.

\section{Objetivos específicos}

a) Determinar la relación directa entre las dimensiones decisión oportuna y gestión institucional en los docentes de la universidad privada Sergio Bernales. 
b) Determinar la relación directa entre las dimensiones análisis de la información y gestión pedagógica en los docentes de la universidad privada Sergio Bernales.

c) Determinar la relación directa entre las dimensiones flexibilidad y adaptación y gestión comunitaria en los docentes de la universidad privada Sergio Bernales.

d) Determinar la relación directa entre las dimensiones evaluación de alternativas y gestión administrativa en los docentes de la universidad privada Sergio Bernales.

\section{Hipótesis}

Existe una relación directa entre la toma de decisiones y la gestión educativa en los docentes de la universidad privada Sergio Bernales.

\section{Hipótesis Específicas}

a) Existe una relación directa entre las dimensiones decisión oportuna y gestión institucional en los docentes de la universidad privada Sergio Bernales.

b) Existe una relación directa entre las dimensiones análisis de información y gestión pedagógica en los docentes de la universidad privada Sergio Bernales.

c) Existe una relación directa entre las dimensiones flexibilidad y adaptación y gestión comunitaria en los docentes de la universidad privada Sergio Bernales.

d) Existe una relación directa entre las dimensiones evaluación de alternativas y gestión administrativa en los docentes de la universidad privada Sergio Bernales.

\section{Importancia}

La investigación es relevante porque en la gestión educativa la toma de decisiones es un factor indispensable en la resolución de problemas, la propuesta de mejoras y otras acciones a ejecutarse en la institución, no solo el líder pedagógico es responsable de la toma de decisiones, sino también los docentes gestores que evaluaran las alternativas de solución proyectándose a alcanzar las metas y objetivos institucionales. Para lograr los objetivos propuestos en la investigación se consideró las bases teóricas de la gestión educativa donde Cassasus (2000) lo define "como la capacidad para articular los recursos del que dispone la organización para lograr lo que se desea" (p.25). Esto significa que el capital intelectual debe tener la capacidad de relacionar sus conocimientos, capacidades, habilidades, estrategias, talento con la estructura del sistema, los recursos disponibles y los objetivos de la organización de tal manera que se genere una conexión directa que lleve a la organización a alcanzar los estándares de calidad. La investigación se fundamenta en el paradigma de la gestión educativa estratégica la cual se sustenta en tres ejes: (a) el pensamiento sistémico, donde se gestiona, organiza y se toma decisiones a nivel institucional, esto conllevará a establecer los objetivos, metas y visión de la escuela. (b) el aprendizaje organizacional, contempla convertir a las instituciones en organizaciones inteligentes a partir de un aprendizaje colectivo del personal y trabajo en equipo. (c) el liderazgo pedagógico, donde el director y los docentes deben promover el cambio institucional a través de un liderazgo transformacional teniendo en cuenta la visión, misión y valores de la organización, todo ello con el propósito de alcanzar la mejora continua de la gestión educativa a partir de una adecuada toma de decisiones y esto se logrará potenciando las capacidades del personal docente. 


\section{Naturaleza y características de la gestión educativa}

De acuerdo con Chadwick (1978) "la gestión educativa como aspecto fundamental de la educación juega un rol importante en la conducción y realización de las actividades, que van a conducir al logro de las metas y objetivos previstos en el sistema educativo" (p. 60). De ahí que se comprende entonces que la gestión educativa puede definirse como el conjunto de actividades y diligencias estratégicas guiadas por procedimientos y técnicas adecuadas para facilitar que las instituciones educativas logren sus metas, objetivos y fines educacionales. Mientras que la administración educativa es el sistema de teorías, categorías y conceptos que describen y explican toda la temática de la organización, conducción y dirección de la educación, la gestión de la educación, es el conjunto de métodos, procedimientos y técnicas que permiten llevar a la práctica la teoría explicativa de la conducción de la educación, en otras palabras, son las estrategias concretas que posibiliten administrar el desarrollo de la educación.

\section{Características de la gestión educativa}

Es un proceso dinamizador; la gestión educativa como conjunto de procedimientos y técnicas, hace posible no solamente la conducción de las empresas e instituciones educativas, sino que también les proporciona, los mecanismos adecuados para su constante cambio de innovación: la gestión es un proceso sistemático; porque los procedimientos, técnicas, instrumentos y estrategias de gestión educativa, constituyen en un conjunto de elementos que están cohesionados entre sí, e interactúan para lograr los propósitos y fines de las instituciones educativas.

Así mismo es un proceso flexible; como sistema de estrategias, métodos y técnicas, posee un alto grado de flexibilidad, en tanto, debe adaptarse a las diversas características sociales y culturales que rodea a la institución educativa. Se sustenta más en la coordinación que en la imposición; la coordinación es una herramienta clave en la conducción y dirección de las instituciones educativas, por tanto, una buena gestión basada en esta estrategia, tendrá definitivamente mayor éxito que aquellas que toman la imposición y la arbitrariedad como práctica permanente.

\section{Enfoques y desarrollo de la gestión educativa}

los siguientes:

Enfoque sistémico: Chadwick (1978) indicó que los elementos del sistema educativo son

a) Estructura: Es el ordenamiento físico o conceptual que conforma el sistema.

b) Entradas: Son elementos que ingresan al sistema procedente del ambiente, conocidos también como los insumos de los cuales se consumen en el proceso o se incorpora al producto.

- Procesos: Son las acciones que se encargan de transformar los insumos y obtener los productos. Estos procesos pueden ser mentales, mecánicos y mental mecánico.

- Salidas: Son el resultados o productos obtenidos del procesamiento o transformación de las entradas que pueden adoptar la forma material (personas o cosas), energía o información. 
- Retroalimentación (feek-back, retroacción o transformación): Consiste en el proceso de control y regulación del sistema mediante el retorno de información, que permite modificar, corregir o cambiar las operaciones con el propósito de obtener productos que respondan a los estándares y patrones de referencia.

- Ambiente: Es el espacio o contexto constituido por objetos y fenómenos, con los que está en permanente interacción, modificándolos o siendo modificado por los agentes educativos.

- La estructura del sistema educativo peruano estaría formada por todos los componentes tanto físicos como abstractos, como niveles, modalidades, programas curriculares, especificaciones de edades, perfiles, etc. que tienen estrecha relación con los fines y objetivos nacionales. En la entrada que comprende los insumos se considera a los docentes, la infraestructura, los alumnos, el currículo, las políticas, el perfil real, los contenidos, etc. Cada uno de estos insumos, interactúan entre ellos para lograr los objetivos educacionales.

El proceso educativo dentro de este enfoque estaría conformado por los procesos académicos o pedagógicos, como: la planificación, la implementación, la organización, la ejecución y la evaluación. En los procesos de gestión administrativa se contempla el proceso de planificación, dirección, ejecución y control. Los egresados con un perfil académico corresponderían a las salidas en el enfoque de este sistema educativo. La retroalimentación son las acciones que se desarrollan después de los resultados de la evaluación de los insumos, procesos, y del producto. Esto tiene como propósito evaluar y reajustar el perfil del egresado en caso de ser necesario. El ambiente seria el contexto social de donde se obtiene los insumos y hacia dónde van los productos.

Enfoque gerencial: Este enfoque se caracteriza por considerar en el desarrollo del proceso educativo a los procesos de gestión, tales como planificación, organización, dirección y control. El desarrollo adecuado de cada uno de estos procesos tiene como propósito lograr los objetivos educacionales previstos. Alvarado (1998) considera como procesos de la gestión: La planificación educativa donde debe considerarse como aspectos relevantes tomar en cuenta: las actividades, los recursos y estrategias utilizadas, con el propósito de alcanzar los objetivos institucionales. Según este enfoque la factibilidad, flexibilidad, objetividad, secuencial, unidad, integral, previsión y la racionalidad deben constituir las bases fundamentales que orienten el trabajo de las instituciones educativas.

Al respecto se conoce que "La dirección es la tarea mediante la cual el administrador se relaciona con sus subalternos para ejercer su autoridad sobre ellas a través de una serie de herramientas o técnicas que son inherentes a quien obtenga un cargo gerencial o directivo". (Alvarado, 1998, p.37). La dirección dentro del enfoque gerencial supone la conducción de la organización, es necesario resaltar la autoridad del director de escuela, o docente gestor quien debe poseer la capacidad de liderazgo, de tal manera que los administrados cumplan sus deberes funcionales. El control en la gestión educativa consiste en evaluar, medir y ponderar los resultados de lo ejecutado, con lo previsto en el plan o programa de desarrollo de la Institución Educativa, pudiendo ejercer dicho control durante todo el proceso y al final del mismo. El propósito fundamental del proceso de control es verificar para corregir, es decir, retroalimentar el proceso si fuera necesario.

\section{Dimensiones de la gestión educativa}


Dimensión institucional: Esta dimensión contribuirá a identificar las formas cómo se organizan los miembros de la Comunidad educativa para el buen funcionamiento de la institución.

Entre estos aspectos se consideran tanto los que pertenecen a la estructura formal (los organigramas, la distribución de tareas y la división del trabajo, el uso del tiempo y de los espacios) como los que conforman la estructura informal (vínculos, formas de relacionarse, y estilos en las prácticas cotidianas, ritos y ceremonias que identifican a la institución) (UNESCO, 2011, p.25).

Esta dimensión ofrece un marco para la sistematización y el análisis de las acciones referidas a aquellos aspectos de estructura que en cada centro educativo dan cuenta de un estilo de funcionamiento. La gestión educativa en su dimensión institucional sostiene:

En la dimensión institucional, es importante promover y valorar el desarrollo de habilidades y capacidades individuales y de grupo, con el fin de que la institución educativa se desarrolle y desenvuelva de manera autónoma, competente y flexible, permitiéndole realizar adaptaciones y transformaciones ante las exigencias y cambios del contexto social (UNESCO,2011, p.24).

Para esto, es necesario tener en claro las políticas institucionales que se desprenden de los principios y la visión que guía a la institución y que se traducirán en formas de hacer en la conducción de la gestión. Por ejemplo, los niveles de participación en la toma de decisiones, si existen o no consejos consultivos, quiénes los conforman y qué responsabilidades asumen, etc.

\section{Dimensión pedagógica:}

Esta dimensión hace referencia al proceso del quehacer educativo que se ejecuta en toda institución y los miembros que la conforman: la enseñanza-aprendizaje. En esta dimensión debemos considerar la labor de los docentes, las prácticas pedagógicas, el dominio en la elaboración de planes y programas, el manejo de los nuevos enfoques pedagógicos y estrategias didácticas, los estilos de enseñanza, las relaciones con los estudiantes, la formación y actualización docente para fortalecer sus competencias profesionales.

"El currículo es una guía para orientar la práctica docente, a partir de la cual se realizarán las actividades de enseñanza y aprendizaje, que explicita y especifica tanto las intenciones educativas como los planes de mejora apropiados para alcanzar esos propósitos" (UNESCO, 2011, p.24).

Dicho de otra manera, el currículum es considerado un instrumento utilizado por los diversos profesionales de la educación: responsables de la administración educativa, docentes, técnicos, especialistas de apoyo a los directores de escuela de la universidad y los supervisores o jefes de áreas entre otros. Que lo utilizan para orientar con el propósito de orientar los procesos de toma de decisiones en relación con la gestión educativa. Vargas (2010) indica que "la gestión 
pedagógica estratégica persigue el cambio cualitativo en la institución a través de la decisión y acción, identifica los problemas, las necesidades de enseñanza y de aprendizaje como debilidades, amenazas, fortalezas y oportunidades asumiendo exigencias de mejoramiento continuo de los procesos pedagógicos" (p.27).

La gestión pedagógica estratégica se caracteriza por: (a) la centralidad en lo pedagógico, (b) habilidades para tratar con lo complejo, (c) el trabajo en equipo, (d) apertura al aprendizaje y a la innovación; (e) asesoramiento y orientación; (f) culturas organizacionales por una visión de futuro, $(\mathrm{g})$ intervenciones sistémicas y estratégicas.

García y Navarro (2007) consideran que en la gestión pedagógica, la dinámica interior de los grupos de trabajo docente promueve el establecimiento de relaciones interpersonales entre docentes y autoridades, entre los grupos de trabajo, favoreciendo el desempeño de la función docente y el cumplimiento de la tarea/producto en el marco de las normas y valores institucionales, a ello se suma al interior de los grupos de trabajo docente, los procesos de comunicación, liderazgo y la toma de decisiones, procesos de la dinámica grupal que permiten definir la percepción del clima institucional (p.32).

\section{Dimensión administrativa:}

De acuerdo con la UNESCO (2011) en el Manual sobre la gestión educativa sostiene:

Esta dimensión busca en todo momento conciliar los intereses individuales con los institucionales, de tal manera que se facilite la toma de decisiones que conlleve a acciones concretas para lograr los objetivos institucionales. Algunas acciones concretas serán la administración del personal, desde el punto de vista laboral, asignación de funciones y evaluación de su desempeño; el mantenimiento y conservación de los bienes muebles e inmuebles; organización de la información y aspectos documentarios de la institución; elaboración de presupuestos y todo el manejo contable-financiero (p.23).

Esta dimensión está constituida por las acciones y estrategias que permiten la conducción del capital humano, los recursos materiales, económicos, procesos técnicos, de tiempo, de seguridad e higiene, por otro lado, el control de la información de los miembros de la institución educativa, el cumplimiento de la normatividad y la supervisión de las funciones, con el propósito de contribuir en la mejora de los procesos de enseñanza-aprendizaje. La adecuada o inadecuada gestión administrativa es uno de los factores que influye en la http://www.monografias.com/trabajos11/veref/veref.shtml eficacia del sistema educativo. Esto significa que la administración es una parte primordial del proceso educativo y constituye el soporte de la gestión pedagógica e institucional en la universidad con el propósito que le permita alcanzar la excelencia y calidad educativa que persigue toda institución.

\section{Dimensión comunitaria:}


"Hace referencia al modo en el que la institución se relaciona con la comunidad de la cual es parte, conociendo y comprendiendo sus condiciones, necesidades y demandas. Asimismo, cómo se integra y participa de la cultura comunitaria" (UNESCO, 2011, p.25). La universidad tiene la responsabilidad de establecer una buena relación con el entorno social e interinstitucional, tomando en cuenta a los grupos de interés (las diversas organizaciones de la comunidad de gestión municipal y regional, estatal, privada, organizaciones civiles, eclesiales y sociedad civil). La participación de los mismos, debe estar orientado a establecer alianzas estratégicas para el mejoramiento de la calidad educativa. Enfatiza en que los docentes universitarios deben estudiar la realidad escolar y establecer lazos con ella, para crear una nueva dinámica de trabajo, en un equipo educativo comunitario. Esta dimensión debe promover una cultura preventiva, que articule la participación de los distintos sectores de la comunidad educativa y especialmente de los estudios en los proyectos y planes institucionales y puedan expresar su punto de vista, en relación con las normas que vayan a presidir la convivencia para prever conflictos.

En el Manual sobre la gestión educativa la UNESCO (2011) al referirse a la dimensión comunitaria, precisa:

Comprende los planes, programas y servicios que la institución pone a disposición de la comunidad, para ofrecer una formación integral con capacidad de valorar y desenvolverse en su entorno social, político, económico y cultural, con el objeto de lograr una mejor calidad de vida, sostenible en el tiempo, para que las futuras generaciones tengan iguales o mejores posibilidades de vida y desarrollo. Hacerse partícipe de la existencia institucional, de sus procesos y decisiones y estimular el apoyo de la familia en relación con el aprendizaje de los estudiantes estableciendo canales de comunicación asertiva (p.28).

\section{Teorías de la variable: toma de decisiones}

"La toma de decisiones es un proceso que se requiere en cualquier actividad, en cualquier situación, desde la más simple hasta la más compleja, la vida consiste en una serie de decisiones y cada una es diferente a las otras pues la experiencia es diferente" (Pareja, 2010, p.29). La toma de decisiones es una acción donde la persona está expuesta en todo momento de su vida y para ello debe contar con los elementos e insumos necesarios que le permita dar las alternativas favorables frente a una situación problemática que presenta la organización. Otros definen "La toma de decisiones como el proceso de aprendizaje natural o estructurado mediante el cual el individuo es capaz de elegir entre dos o más alternativas, para resolver situaciones o conflictos de su vida, entorno familiar y profesional" (Bentacourt,2006, p.47).

Si el tomar una decisión nos conduce a tener dos alternativas; decidirnos por una solución o simplemente dejar que las cosas sucedan sin intervenir para que los resultados muchas veces no sean los esperados. Tomamos decisiones para alcanzar el éxito y por otro lado evitamos algunas veces hacerlo por temor al fracaso. Es necesario entender que es un proceso interno propio del ser humano y que factores emocionales pueden influir en la adecuada o inadecuada elección de alternativas de solución. Los problemas más comunes que influyen en la toma de decisiones para José Betancourt son: (a) Impuntualidad. (b) Conexiones no detectadas con otros problemas. (c) Incumplimiento de las secuencias para decidir adecuadamente. (d) No hacer 
seguimiento a las decisiones. (e) No establecer reglas claras de dirección. (f)Tomar la decisión reactivamente porque se acabó el tiempo. (g) Parálisis del tomador de decisiones. (h) Inseguridad y falta de confianza. (i) Exceso de confianza que lo lleva a uno a creer que las cosas no le pasan a uno. (j) Falta de claridad personal. (k) Indefinición personal. (l) Temores desmedidos.

\section{Los pasos para tomar decisiones}

Para Betancourt (2006), los pasos a considerar en la toma de decisiones son: Establezca con claridad que es lo que realmente desea obtener. Para ello: (a) establezca objetivos, (b) actúe con sinceridad, (c) actúe con integridad, (d) visualice la situación como una oportunidad y no como una amenaza, (e) identifique todos los elementos que le alejan de la decisión adecuada.

Asuma una situación de un fuerte dolor al no tomar una decisión en el momento y un gran placer al tomar la decisión en ese momento. Interrumpa las pautas de pensamiento, sentimiento y acción que lo limitan a actuar de manera adecuada. La decisión que se tome para resolver una situación, debe ser tomada en el mismo nivel de pensamiento en que se generó dicha situación. Por otro lado, tener presente que los modelos mentales y las creencias influyen en la toma de decisiones. Cree nuevas alternativas que sean realmente capacitadoras. Es decir, busque alternativas que lo acerquen al placer o el éxito y lo alejen del dolor o fracaso para ello utilice su creatividad para generar alternativas capacitadoras, las cuales deben seguir una secuencia de: (a) descongelar, implica romper el hielo para retirar los obstáculos que puedan dañar la creatividad, (b) acumular, es reunir y recopilar información aportando nuevos datos, (c) deliberar, es analizar y discutir los datos estratificando la información, (d) incubar, es proporcionar el tiempo y el espacio para que la información fluya, (e) iluminar, es identificar posibles soluciones y alternativas, (f) finalizar, es tomar la decisión.

Condicione sus nuevas pautas de acción, hasta lograr que sean consistentes. Asegúrese del éxito de la decisión a largo plazo reforzando la decisión. Ponga a prueba sus decisiones, la comprobación implica realizar una revisión de su decisión en el sistema, visualizar los posibles efectos de su decisión a corto, mediano y largo plazo.

\section{Cualidades personales en la toma de decisiones}

Estas cualidades son inherentes al individuo al momento de tomar decisiones: (a) experiencia, es importante en el sentido que el líder cuando se enfrenta a un problema toma en cuenta el tiempo en el cargo desempeñado y otras situaciones similares por las que ha tenido que sobrellevar y tomar decisiones. (b) buen juicio, es la forma como el individuo evalúa de manera inteligente la información que le permitirá tomar decisiones. Esta habilidad esta reforzada por la experiencia, la madurez y el razonamiento. Un juicio se basa en la información disponible los hechos, las opiniones, el conocimiento en general y su experiencia anterior. (c) creatividad, es una habilidad del tomador de decisiones asociada con la capacidad de formular ideas innovadoras, proponer alternativas únicas para dar solución inmediata a situaciones conflictivas del momento. (d) habilidades cuantitativas, es la habilidad de emplear técnicas, métodos cuantitativos o investigación de operaciones. Estas herramientas proporcionan a los líderes a tomar decisiones efectivas. Sin embargo, no olvidar que el juicio no puede ser remplazado por esta habilidad. 


\section{Etapas de la toma de decisiones}

Para Betancourt (2006) estas etapas a considerarse son:

(a) Identificación y diagnóstico del problema, se reconoce el problema que deseamos solucionar, partiendo por un diagnóstico de la situación para luego proponer las medidas a tomar.

(b) Generación de soluciones alternativas, se trata de proponer una variedad de soluciones creativas e innovadoras, las cuales se sugieren deben ser planteadas teniendo en cuenta la certidumbre en la información.

(c) Selección de la mejor alternativa, el líder debe proyectarse a considerar los posibles efectos de sus opciones. Antes de tomar la decisión debe considerar maximizar, satisfacer y optimizar sus opciones al momento de elegir la mejor alternativa.

(d) Evaluación de alternativas, el líder debe predecir los posibles efectos y consecuencias de cada una de sus alternativas u opciones planteadas con el propósito de tomar decisiones acertadas. Por otro lado, es importante contar con planes de contingencia en la organización en caso de una inadecuada decisión tomada por el mando a cargo.

(e) Evaluación de la decisión, es primordial porque permitirá evaluar los resultados obtenidos y a partir de ellos plantearse una retroalimentación del proceso, si esta es positiva es posible continuar sin problemas e incluso se podría aplicar la misma decisión a otras áreas de la organización. Por el contrario, si la retroalimentación es negativa es necesario contar con el tiempo, recursos, esfuerzos o pensamiento para volver al principio del proceso. (p.58)

Implementación de la decisión, una vez tomada la decisión debe ser implementada y el personal debe sentirse involucrado para poder participar de estas decisiones, aceptando los cambios. Debe existir la comprensión total sobre de su implementación exitosa. Para tal fin, las personas que participan en esta fase del proceso, deberían estar involucradas desde las primeras etapas que anteriormente hemos mencionado.

\section{Características de la toma de decisiones en las Instituciones educativas}

Analizando las características de la toma de decisiones Emily (2011) refiere "las organizaciones tienen normas y regulaciones relacionadas con un proceso por medio del cual un gerente puede llegar a alcanzar objetivos, políticas y estrategias" (p.12). Por ello se establecen cinco características fundamentales:

(i) Efectos futuros, En lo que se refiere a este punto podemos definir que los compromisos relacionados con la decisión afectarán el futuro de una institución. Un objetivo programado para el futuro implica una decisión a largo plazo, puede ser considerada una decisión de alto nivel, mientras que una decisión con efectos a corto plazo puede ser tomada a un nivel muy inferior.

(ii) Reversibilidad, Al analizar esta característica, la misma que se refiere a la velocidad con que una decisión puede revertirse y la dificultad que implica hacer este cambio. Se puede establecer que revertir es difícil, es más recomendable tomar la decisión a un nivel alto; pero si revertir es fácil, se requiere tomar la decisión a un nivel bajo. 
(iii) Impacto, La toma de decisiones necesariamente implica involucrar otras áreas o actividades que se ven afectadas. Si el impacto es amplio, es indicado tomar la decisión a un nivel superior; un impacto único se asocia con una decisión tomada a un nivel inferior.

(iv) Calidad, Si la calidad es alta involucra, valores éticos, consideraciones legales, principios básicos de conducta, imagen de la compañía, etc. Estos factores requieren tomar luna decisión a un nivel alto; si solo algunos factores son relevantes, se recomienda tomar a decisión a un nivel bajo.

(v) Periodicidad, Si la toma de decisiones se considera excepcional y frecuente, en consecuencia, estas tienen que ser en alto y bajo nivel. Una decisión excepcional es una decisión de alto nivel, mientras que una decisión que se toma frecuentemente es una decisión de nivel bajo.

Según las características se puede apreciar que los efectos futuros son relevantes en una toma de decisiones, el impacto tiene que ser inmediato de acuerdo en el nivel en que se da. Asimismo, para no afectar la calidad sin romper relaciones interpersonales se tomará la decisión a un nivel alto. La toma de decisiones debe ser inmediata y oportuna.

\section{Dimensiones de la toma de decisiones}

Betancourt (2006, p. 45) considera que son las cualidades que tienen las personas que toman decisiones apegada a las circunstancias en que se dan y difieren en cuanto a la utilización de la información y cómo crear alternativas de solución. Se distinguen:

Dimensión Decisión oportuna: Betancourt (2006, p. 46) sostiene que "es la cualidad donde el individuo toma la decisión de forma rápida, eficiente, firme a partir de escasa información y evalúan pocas alternativas de solución, sin embargo, valora la acción porque una vez fijado el plan se compromete y se preocupa por cumplirlo. Estas personas se caracterizan por valorar la lealtad, honestidad y claridad. Además de ser eficientes y lógicos”.

Dimensión Flexibilidad y adaptación: Betancourt (2006, p. 46) sostiene que "es la cualidad de la persona que toma decisiones rápidas sin demorar en el análisis de la información y decide sobre diversas alternativas, es por ello que este estilo se caracteriza por ser rápido y adaptable es decir cambiante de acuerdo a las circunstancias y la variedad de alternativas con las que cuenta para decidir. Los individuos con este estilo son receptivos y muy sociables que valora la información justa y veraz".

Dimensión Análisis de información: Betancourt (2006, p. 47) precisa que "es la cualidad en que se toman decisiones a partir del manejo de mucha información, manteniendo una posición firme en cuanto a su decisión final resistiéndose a cualquier manifestación de cambio en el tiempo. Los individuos se caracterizan por ser altamente intelectuales".

Dimensión Evaluación de alternativas: Betancourt (2006) precisa que “es la cualidad donde antes de decidir trata de conocer variada información y evaluar múltiples alternativas les permitirá tomar decisiones acertadas, pero sin embargo estas pueden cambiar con el tiempo y las circunstancias. Estas personas se caracterizan por ser muy participativas y creativas" (p. 48). 


\section{La información clave en la toma de decisiones}

Este recurso y su tratamiento determinan la efectividad del proceso tanto para el desarrollo de este, como para la información que se requiere la misma que debe ser precisa, oportuna, fidedigna y suficiente para tomar la mejor decisión posible si obviamos este tipo de información, el individuo no puede percibir adecuadamente la situación problemática y tampoco podría valorar alternativas de solución porque tendría la información necesaria para tomarla, ni los recursos para su adecuado tratamiento. De acuerdo con Bernal (2006) además de estos componentes podemos considerar otros elementos contextuales: la gestión organizacional, la cultura organizacional e informacional, la alfabetización informacional como parte de esta última, el aprendizaje organizacional, las nuevas tecnologías de información y comunicaciones, el proceso de comunicación organizacional y las políticas y normas institucionales (p. 41).

Los ingredientes para la toma de decisiones se encuentran:

(i) Información: En lo que se refiere a la información, la decisión debe basarse en datos disponibles.

(ii) Conocimientos: El conocimiento es fundamental porque si se conocen las circunstancias que rodean al problema, entonces se puede seleccionar un curso de acción favorable.

(iii) Experiencia: La experiencia proporciona la información necesaria para resolver un problema futuro similar.

(iv) Análisis: Los métodos para el análisis en la toma de decisiones son determinantes.

(v) Juicio: Es necesario para combinar la información, los conocimientos, la experiencia y el análisis, con el fin de seleccionar un curso de acción adecuado.

A rasgos generales, las organizaciones tienden a evaluar el proceso de toma de decisiones y valorar la efectividad de las decisiones que toman. Sin embargo, este proceso se puede valorar a la vez por el comportamiento de sus componentes y el desarrollo de sus procedimientos. También es importante destacar que la toma de decisiones está condicionada por múltiples factores, entre los que se encuentran los factores mencionados anteriormente.

\section{Condiciones para la toma de decisiones}

Robbins precisa que al tomar las decisiones un gerente debe enfrentar estas condiciones:

Certidumbre; es una situación ideal para la toma de decisiones "un gerente puede tomar decisiones precisas debido a que conoce el resultado de cada alternativa" (Robbins, 2010 p.129). En esta situación el individuo conoce y está bien informado del problema, las alternativas de solución son evidentes, esto le permite prever y controlar los resultados de su toma de decisiones. Asimismo, es capaz de decidir por la solución acertada y el resultado más óptimo.

Riesgo; es la situación más común que se presenta porque "son condiciones en el que el tomador de decisiones puede estimar la probabilidad de ciertos resultados. En situaciones de riesgo los gerentes tienen información histórica de experiencias personales o información secundaria que les permite asignar probabilidades a diferentes alternativas." (Robbins, 2010, p.129). El riesgo significa que el individuo quien toma las decisiones puede equivocarse al 
decidir entre una alternativa u otra, esta toma de decisiones equivocadas lo lleva a obtener resultados y consecuencias negativas para la organización. Esta probabilidad es alta cuando la incertidumbre de una situación problemática es evidente.

Incertidumbre; en estas condiciones, "La elección de alternativas se ve influenciada por la cantidad limitada de información disponible y por la orientación psicológica del tomador de decisiones". Con frecuencia la incertidumbre obliga a los gerentes a confiar en su intuición, corazonadas, creatividad y presentimientos. (Robbins, 2010, p.130) El individuo no cuenta con la suficiente información o la situación problemática y las posibles alternativas son ambiguas, esto significa un grave riesgo al momento de decidirse. Esta condición puede presentarse en la vida personal y profesional cuando se inician proyectos nuevos.

\section{Método}

El tipo de investigación es bàsica, nivel descriptivo correccional, el diseño de investigación es no experimental y corte transversal.

\section{La población y muestra}

La población de estudio está constituida por 84 docentes de las carreras de ingeniería de sistemas, obstetricia; y derecho y ciencia política de la universidad privada Sergio Bernales, ubicado en la provincia de Cañete, departamento de Lima. La muestra lo constituyo 42 docentes universitarios de las tres carreras profesionales ya mencionados.

\section{Tabla 1: Distribución de la población}

\begin{tabular}{lccc}
\hline Nivel & \multicolumn{2}{c}{ Docentes universitarios } & Total \\
\cline { 2 - 3 } & $\mathrm{H}$ & $\mathrm{M}$ & \\
Ingeniería de Sistemas & 05 & 01 & 06 \\
obstetricia & 08 & 30 & 38 \\
derecho y ciencia política & 33 & 07 & 40 \\
Total & 46 & 38 & 84 \\
\hline
\end{tabular}

\section{Métodos de análisis de datos}

La técnica utilizada para la obtención de la información es la observación a partir del instrumento lista de cotejo que contiene ítems sobre los indicadores y dimensiones de la variable dependiente toma de desiciones que fue aplicada al personal docente de la universidad privada Sergio Bernales de la provincia de Cañete, Lima. Es importante mencionar que el instrumento tiene un nivel de confiabilidad aceptable con un valor de 0.87 según la prueba del coeficiente de confiabilidad alfa de cronbach. Después de la recogida de datos se procesó la información mediante el sistema SPSS y los estadísticos de la media aritmética y la desviación estándar. La prueba estadística seleccionada para probar la hipótesis fue la correlación de Pearson.

\section{Resultados}

Los resultados obtenidos luego de aplicar la lista de cotejo, y analizar los resultados con la correlacion de Pearson, se concluye que si existe una correlación significativa entre las 
dimesniones de la variable gestión educativa con la toma de desiciones de los directivos o docentes gestores de la universidad privada Sergio Bernales.

Tabla 2. Nivel de correlación de la variable toma de desiciones con la dimensión gestión institucional

\begin{tabular}{llrr}
\hline & \multicolumn{2}{c}{ Correlaciones } \\
\hline \multirow{2}{*}{ TOMA DE DECISIONES } & TOMA DE DECISIONES & Gestión institucional \\
& Correlación de Pearson & 1 &, 795 \\
& Sig. (bilateral) &, 038 \\
Gestión institucional & N & 42 & 42 \\
& Correlación de Pearson &, 795 & 1 \\
& Sig. (bilateral) & 0,038 & 42 \\
& N & 42 & 4 \\
\hline
\end{tabular}

Fuente: base de datos

De acuerdo con la información recogida y organizada en la tabla 2, al aplicar la lista de cotejo a la muestra se evidencia que si hay una correlación entre la toma de decisiones y la gestión institucional con una significancia de $0,038(\mathrm{p}<0,05)$ y que la correlación entre ambas tiene un nivel altamente positivo en una muestra de 42.

Tabla 3. Nivel de correlación de la variable toma de desiciones con la dimensión gestión pedagògica

\begin{tabular}{|c|c|c|c|}
\hline \multicolumn{4}{|c|}{ Correlaciones } \\
\hline & & $\begin{array}{c}\text { TOMA DE } \\
\text { DECISIONES }\end{array}$ & $\begin{array}{c}\text { Gestión } \\
\text { pedagógica }\end{array}$ \\
\hline \multirow{4}{*}{$\begin{array}{c}\text { TOMA DE } \\
\text { DECISIONES }\end{array}$} & Correlación de & - &,- 790 \\
\hline & Pearson & & \\
\hline & Sig. (bilateral) & &, 050 \\
\hline & $\mathrm{N}$ & 42 & 42 \\
\hline \multirow[t]{4}{*}{ Gestión pedagógica } & Correlación de &,- 790 & 1 \\
\hline & Pearson & & \\
\hline & Sig. (bilateral) &, 050 & \\
\hline & $\mathrm{N}$ & 42 & 42 \\
\hline
\end{tabular}

Fuente: base de datos

De acuerdo con la información recogida y organizada en la tabla 3, al aplicar la lista de cotejo a la muestra se tiene como resultado que si existe relación entre la toma de decisiones y la gestión pedagógica $(\mathrm{p}<=0,05)$ y que la correlación es altamente negativa puesto que el valor del coeficiente de Pearson es -790 en una muestra de 42.

Tabla 4. Nivel de correlación de la variable toma de desiciones con la dimensión gestión

\begin{tabular}{cccr}
\multicolumn{3}{c}{ comunitaria } \\
\hline & Correlaciones & \\
\hline & TOMA DE & Gestión \\
TOMA DE & DECISIONES & comunitaria \\
DECISIONES & Correlación de & 1 &, 917 \\
& Pearson & &, 000 \\
& Sig. (bilateral) & & 42 \\
\hline
\end{tabular}




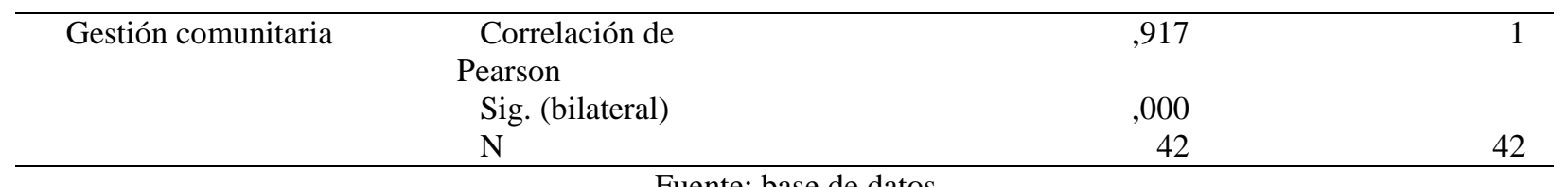

Fuente: base de datos

De acuerdo con la información recogida y organizada en la tabla 4, al aplicar la lista de cotejo a la muestra se tiene como resultado que existe un alto nivel de correlación entre la toma de decisiones y la gestión comunitaria $(\mathrm{R}=0,917)$ con una significancia de 0,000 .

Tabla 5. Nivel de correlación de la variable toma de desiciones con la dimensión gestión administrativa

\begin{tabular}{|c|c|c|c|}
\hline \multicolumn{4}{|c|}{ Correlaciones } \\
\hline & & $\begin{array}{c}\text { TOMA DE } \\
\text { DECISIONES }\end{array}$ & $\begin{array}{c}\text { Gestión } \\
\text { administrativa }\end{array}$ \\
\hline \multirow{4}{*}{$\begin{array}{c}\text { TOMA DE } \\
\text { DECISIONES }\end{array}$} & Correlación de & 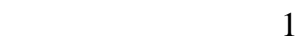 &, $680^{* *}$ \\
\hline & Pearson & & \\
\hline & Sig. (bilateral) & & ,027 \\
\hline & $\mathrm{N}$ & 42 & 42 \\
\hline \multirow[t]{3}{*}{ Gestión administrativa } & $\begin{array}{l}\text { Correlación de } \\
\text { Pearson }\end{array}$ &, $680^{* *}$ & 1 \\
\hline & Sig. (bilateral) & ,027 & \\
\hline & $\mathrm{N}$ & 42 & 42 \\
\hline *. La correlación es sig & ativa en el nivel 0 , & & \\
\hline
\end{tabular}

Fuente: base de datos

En la tabla 5 se observa que el valor de p (sig. bilateral) es menor a 0,05; por tanto, eso nos evidencia que si existe correlación entre la toma de decisiones y la gestión administrativa y que además la correlación entre ambas es alta puesto que el valor del coeficiente de correlación de Pearson es 0,680.

\section{Discusión}

Para lograr una óptima gestión educativa es necesario que sus dimensiones gestión institucional, pedagógica, comunitaria y administrativa se desarrollen en una adecuada toma de desiciones que permitan que la institución alcance sus metas y objetivos institucionales. En la Universidad privada Sergio Bernales se evidencio dificultades en su gestión educativa por no encontrarse una adecuada toma de decisiones, motivo de la investigación para analizar su propósito que superar las limitaciones en este contexto. El instrumento utilizado fue la lista de cotejo en el sé que presenta una buena confiabilidad y alta consistencia lo que recomienda su aplicación en la muestra de estudio. La variable independiente presenta una significativa influencia sobre la variable dependiente debido a la ejecución de acciones y estrategias adecuadas. Sobre la relación entre la toma de desiciones y la gestión educativa podemos precisar que existe correlación en especial en las dimensiones institucionales, comunitarias y administrativas, y en menor grado en la dimensión pedagógica; evidenciada despues de la aplicación de la lista de cotejo en el recojo de datos. Los resultados coinciden con los resultados de García y Navarro (2007) consideran que, en la gestión pedagógica, permite la dinámica interior de los grupos de trabajo docente y promueve el establecimiento de relaciones interpersonales entre docentes y autoridades en los grupos de trabajo. 


\section{Conclusiones}

Con respecto al objetivo general se concluye que existe relación significativa entre la variable toma de desiciones y la variable gestión eductaiva. En referencia a los objetivos específicos se concluye que existe relación de la toma de desiciones de los directores de escuela y jefes de área asumidos por los docentes gestores tiene relación significativa con dimensión gestión pedagógica, seguida de la dimensión gestión institucional, la gestión comunitaria y administrativa y que ello contribuye en el logro de los objetivos institucionales para la mejora de la calidad del servicio en la universidad privada Sergio Bernales.

De acuerdo a los resultados generales obtenidos con el estadístico de la correlación de Pearson la variable toma de decisiones presentó un valor .491 de grado de correlación con la variable gestión educativa analizado en sus dimensiones gestión institucional, pedagógica, comunitaria, y administrativa siendo sus valores significativos. Por lo que se concluye que la toma de decisiones es desiciva para una adecuada gestión educativa, en la universidad privada Sergio Bernales, 2017; siendo mayor la correlacion en la dimensión gestión institucional y gestión administrativa, y en la dimensión pedagógica y comunitaria poco significativa.

\section{Bibliografía}

Alvarado, O. (1999). Getiòn Educativa.Enfoques y procesos. Lima : Editorial Lima.

Bentacourt, J. R. (2006). Toma de decisiones :Obtener el éxito. De gerencia.com.

Bernal, C. (2000). Metodologìa de la investigaciòn II. Lima: Pearson.

Botero, C. (2009). Cinco tendencias de la gestiòn educativa. Revista Iberoamericana de Educaciòn, 49.

Casassus, J. (2000). Problemas de la gestiòn educativa en Amèrica Latina. UNESCO.

Chadwick, C. (2006). Tecnologìa educacional para el docente. Madrid, España: Paidòs-Ibèrica S.A.

Chiavenato, I. (2006). Introducciòn a la teorìa general de la administraciòn. Mc Graw Hill Interamericana.

Chiavenato, I. (2007). Administraciòn de recursos humanos.El capital humano de las organizaciones . Mc Graw Hill Interamericana.

Delgado, S. (2011). El mejoramiento de la gestiòn educativa a travès de procesos de calidad debe bajar los indices de deserciòn en el colegio de la enseñanza Cardenal Luque, compañia de Marìa en Bogotà. Colombia: Tesis de grado.Universidad de Granada.

Delors, J. (1996). La educaciòn encierra un tesoro. Madrid: Informe a la UNESCO de la comisiòn internacional de educaciòn para el siglo XXI.

Diccionario de las Ciencias de la Educaciòn. (1998). España: Editorial Santillana. 
Edvinsson, L. (1999). El capital intelectual:como identificar y calcular el valor de los recursos intangibles de su empresa. España.

Elera, R. (2010). Gestiòn institucional y su relaciòn con la calidad del servicio en una instituciòn educativa pùblica del Callao. Lima: Tesis de grado. Universidad San Ignacio de Loyola.

Emily, M. (2011). Toma de decisiones . Comunidad educativa mundial ilustrados.

Gallegos, J. (2004). Gestiòn educativa en el proceso de descentralizaciòn. Lima: San Marcos.

Garcìa, A., \& Navarro, R. (2007). Clima y compromiso organizacional. Lima.

Gutiérrez, H. G. (2014). Teoría de la toma de decisiones. Definición, etapas y tipos. Fonte: http://gestiopolis.com/teoría-de-la.-toma-de-decisiones-definicion-etapas-y-tipos/

Hernàndez, R. (2010). Metodologìa dela investigaciòn. México: Mc Graw Hill.

Ley general de educaciòn. (1999). Abedul. Lima.

López, M. (2012). Gerencia: capital intelectual y sus competencias en instituciones educativas. Fonte: http://servicio.bc.uc.ve/faces/revista/lainet/lainetv5n9/art05.pdf

Mèndez, P. (2012). Autoevaluaciòn de la calidad de gestiòn en una instituciòn educativa de VentanillaCallao. Lima: Tesis de grado.Universidad San Ignacio de Loyola.

UNESCO. (2011). Manual de gestiòn para directores de instituciones educativas. Lima: Gestiòn institucional.

OREALC/UNESCO. (2007). El derecho a una educaciòn de calidad para todos en Amèrica Latina y el Caribe. Revista electrònica iberoamericana sobre calidad, eficacia y cambio en educaciòn., 29-35.

Rentería, N. Q. (2010). Diseño de una estrategia de gestión educativa para mejorar los niveles de Convivencia en el colegio Rafael Uribe. Colombia: Tesis de grado. Pontificia Universidad Javeriana.

Robbins, S. (2002). Fundamentos del comportamiento organizacional. España: Editorial Prentice-Hall Hispanoamericana.

Sànchez, C. (2002). Metodologìa y diseños en la investigaciòn cinentifica . Lima: Editorial Universitaria.

Vargas, D. (2010). Gestiòn pedagògica del trabajo docente a travès de grupos cooperativos. Lima: Tesis de grado.Universidad Catòlica del Perù.

Vílchez, G. (2014). Evaluación de la gestión educativa del centro de educación técnico productivo Madre Admirable . Lima: Tesis de grado. Universidad católica del Perú. 